\title{
Total Graph Interpretation of the Numbers of the Fibonacci Type
}

\section{Urszula Bednarz, Iwona Włoch, and Małgorzata Wołowiec-Musiał}

Faculty of Mathematics and Applied Physics, Rzeszów University of Technology, Aleja Powstańców Warszawy 12, 35-359 Rzeszów, Poland

Correspondence should be addressed to Urszula Bednarz; ubednarz@prz.edu.pl

Received 7 May 2015; Revised 14 September 2015; Accepted 21 September 2015

Academic Editor: Frank Werner

Copyright (C) 2015 Urszula Bednarz et al. This is an open access article distributed under the Creative Commons Attribution License, which permits unrestricted use, distribution, and reproduction in any medium, provided the original work is properly cited.

We give a total graph interpretation of the numbers of the Fibonacci type. This graph interpretation relates to an edge colouring by monochromatic paths in graphs. We will show that it works for almost all numbers of the Fibonacci type. Moreover, we give the lower bound and the upper bound for the number of all $\left(A_{1}, 2 A_{1}\right)$-edge colourings in trees.

\section{Introduction and Preliminary Results}

For general concepts about combinatory graph theory and online encyclopedia of integer sequences, see, for example, [1], [2], and [3], respectively. By numbers of the Fibonacci type we mean numbers defined recursively by the $k$ th-order linear recurrence relation of the form

$$
a_{n}=b_{1} a_{n-1}+b_{2} a_{n-2}+\cdots+b_{k} a_{n-k}
$$

for $n \geq k$, where $k \geq 2$ and $b_{i} \geq 0, i=1, \ldots, k$, are integers and $a_{0}, \ldots, a_{k-1}$ are given integers. For special values of $k$ and $b_{i}$, $i=1, \ldots, k$, the equality (1) gives the well-known recurrences which define the numbers of the Fibonacci type. They are listed below.

(a) Fibonacci numbers $F_{n}$ :

$$
F_{n}=F_{n-1}+F_{n-2}, \quad n \geq 2
$$

with $F_{0}=F_{1}=1$.

(b) Lucas numbers $L_{n}$ :

$$
L_{n}=L_{n-1}+L_{n-2}, \quad n \geq 2
$$

with $L_{0}=2, L_{1}=1$. (c) Pell numbers $P_{n}$ :

$$
P_{n}=2 P_{n-1}+P_{n-2}, \quad n \geq 2
$$

with $P_{0}=0, P_{1}=1$.

(d) Pell-Lucas numbers $Q_{n}$ :

$$
Q_{n}=2 P_{n-1}+P_{n-2}, \quad n \geq 2
$$

with $P_{0}=P_{1}=1$.

(e) Jacobsthal numbers $J_{n}$ :

$$
J_{n}=J_{n-1}+2 J_{n-2}, \quad n \geq 2
$$

with $J_{0}=0, J_{1}=1$.

(f) Jacobsthal-Lucas numbers $j_{n}$ :

$$
j_{n}=j_{n-1}+2 j_{n-2}, \quad n \geq 2
$$

with $j_{0}=2, j_{1}=1$.

(g) Padovan numbers $\operatorname{Pv}(n)$ :

$$
\operatorname{Pv}(n)=\operatorname{Pv}(n-2)+\operatorname{Pv}(n-3), \quad n \geq 3
$$

with $\operatorname{Pv}(0)=\operatorname{Pv}(1)=\operatorname{Pv}(2)=1$. 
(h) Perrin numbers $\operatorname{Pr}(n)$ :

$$
\begin{aligned}
& \operatorname{Pr}(n)=\operatorname{Pr}(n-2)+\operatorname{Pr}(n-3), \quad n \geq 3 \\
& \text { with } \operatorname{Pr}(0)=3, \operatorname{Pr}(1)=0, \operatorname{Pr}(2)=2 \text {. }
\end{aligned}
$$

(i) Tribonacci numbers of the first kind $T_{n}$ :

$$
T_{n}=T_{n-1}+T_{n-2}+T_{n-3}, \quad n \geq 3
$$

with $T_{0}=T_{1}=T_{2}=1$.

(j) Tribonacci numbers of the second $\operatorname{kind} T_{n}^{*}$ :

$$
T_{n}^{*}=T_{n-1}^{*}+T_{n-2}^{*}+T_{n-3}^{*}, \quad n \geq 3
$$

with $T_{0}^{*}=0, T_{1}^{*}=T_{2}^{*}=1$.

These numbers are intensively studied in the literature; they have many interesting interpretations also in graphs. The graph interpretation of the Fibonacci numbers was initiated by Prodinger and Tichy in [4]. In that paper, among others, they showed connections between the Fibonacci and the Lucas numbers and the number of all independent sets in special graphs. Let $\mathrm{NI}(G)$ be the number of all independent sets in a graph $G$. By $\mathbb{P}(m), \mathbb{C}(m), \mathbb{T}(m)$, and $\mathbb{S}(m)$ we denote a path, a cycle, a tree, and a star of size $m$ for $m \geq 1$, respectively. Then, $\operatorname{NI}(\mathbb{P}(m))=F_{m+2}$ and $\operatorname{NI}(\mathbb{C}(m))=L_{m}$; for details, see [4]. This simple observation gave an impetus for studying the graph parameter $\mathrm{NI}(G)$ in different classes of graphs and their products, from the pure mathematical point of view. This interest was multiplied by the fact that the parameter $\mathrm{NI}(G)$ of a molecular graph was introduced to the combinatorial chemistry by showing some relations between $\mathrm{NI}(G)$ and some physicochemical properties of chemical compounds. For these reasons, the parameter $\mathrm{NI}(G)$ is intensively studied in graphs.

Theorem 1 (see [4]). Let $m \geq 1$ be an integer. For a tree $\mathbb{T}(m)$ of size $m, F_{m+2} \leq N I(\mathbb{T}(m)) \leq 2^{m}+1$ holds. Moreover, $N I(\mathbb{T}(m))=F_{m+2}$ iff $\mathbb{T}(m)=\mathbb{P}(m)$ and $N I(\mathbb{T}(m))=2^{m}+1$ iff $\mathbb{T}(m)=\mathbb{S}(m)$.

The graph parameter $\mathrm{NI}(G)$ relates to other numbers of the Fibonacci type. For Jacobsthal numbers $J_{n}$ and JacobsthalLucas numbers $j_{n}$, the following has been proved.

Theorem 2 (see $[5,6])$. Let $m \geq 1$ be an integer. Then, $N I\left(\mathbb{P}(m)\left[K_{2}\right]\right)=J_{m+3}$ and $N I\left(\mathbb{C}(m)\left[K_{2}\right]\right)=j_{m}$.

For other results related to the parameter $\mathrm{NI}(G)$ and their applications, see the last survey [7]. In this survey the authors collect and classify the results concerning the graph parameter $\mathrm{NI}(G)$, most of which are obtained quite recently. Actually for the chemical applications, the index $\mathrm{NI}(G)$ is named as the Merrifield-Simmons index.

Considering the Fibonacci numbers and numbers of the Fibonacci type, we can collect other graph parameters related to the numbers of the Fibonacci type.

Let $Z(G)$ be the number of all matchings of $G$. Then, $Z\left(\mathbb{P}(m) \circ K_{1}\right)=P_{m+2}$ and $Z\left(\mathbb{C}(m) \circ K_{1}\right)=2 Q_{m}$, where
$G \circ H$ is the corona of two graphs. The index $Z(G)$ is wellknown by the Hosoya index. Let $\mathrm{NI}_{L}(G)$ be the number of all independent sets in $G$ including the set of leaves as a subset. Then, $\mathrm{NI}_{L}(\mathbb{P}(m))=\operatorname{Pv}(m-2)$. For this graph parameter, see more details in [8].

Theorem 3 (see [8]). Let $m \geq 2$ be an integer. Then, for a tree $\mathbb{T}(m)$ of size $m, N I_{L}(\mathbb{T}(m)) \leq P v(m-2)$ holds. Moreover, $\mathbb{P}(m)$ is the extremal graph achieving the maximum value of $N I_{L}(\mathbb{T}(m))$.

For the classical Fibonacci numbers and numbers of the Fibonacci type, there are many generalizations with respect to one or more parameters. We list some of these generalized numbers of the Fibonacci type. Let $k$ and $n$ be integers. We have the following.

(1) $k$-generalized Fibonacci numbers $f_{n}$ (Miles Jr. [9]):

$$
f_{n}=f_{n-1}+f_{n-2}+\cdots+f_{n-k}, \quad k \geq 2, n>k,
$$

with $f_{j}=0$ for $0 \leq j \leq k-2$ and $f_{k-1}=f_{k}=1$.

(2) Generalized Fibonacci numbers $F(k, n)$ (Kwaśnik and Włoch [10]):

$F(k, n)=F(k, n-1)+F(k, n-k), \quad k \geq 2, n \geq k$,

with $F(k, n)=n+1$ for $n=0,1, \ldots, k-1$.

(3) $k$-Fibonacci numbers $F_{k}(n)$ (Falcón and Plaza [11]):

$F_{k}(n)=k F_{k}(n-1)+F_{k}(n-2), \quad k \geq 1, n \geq 2$,

with $F_{k}(0)=0$ and $F_{k}(1)=1$.

(4) Generalized Pell numbers $P(k, n)$ (Włoch [12]):

$$
\begin{aligned}
P(k, n)= & P(k, n-1)+P(k, n-k+1) \\
& +P(k, n-k), \quad k \geq 2, n \geq k+1,
\end{aligned}
$$

with $P(2,0)=0$ and $P(k, 0)=1$ for $k \geq 3$ and $P(k, 1)=1, P(k, n)=2 n-2$ for $2 \leq n \leq k$.

(5) $k$-Lucas numbers $L_{k}(n)$ (Falcon [13]):

$$
\begin{aligned}
& L_{k}(n)=k L_{k}(n-1)+L_{k}(n-2), \quad k \geq 1, n \geq 2, \\
& \quad \text { with } L_{k}(0)=2 \text { and } L_{k}(1)=k .
\end{aligned}
$$

(6) $k$-Pell numbers $P_{k}(n)$ (Catarino [14]):

$$
\begin{aligned}
& P_{k}(n)=2 P_{k}(n-1)+k P_{k}(n-2), \quad k \geq 1, n \geq 2, \\
& \quad \text { with } P_{k}(0)=0 \text { and } P_{k}(1)=1 .
\end{aligned}
$$

(7) $k$-Pell-Lucas numbers $Q_{k}(n)$ (Catarino and Vasco [15]):

$$
\begin{aligned}
& Q_{k}(n)=2 Q_{k}(n-1)+k Q_{k}(n-2), \quad k \geq 1, n \geq 2, \\
& \text { with } Q_{k}(0)=Q_{k}(1)=2 .
\end{aligned}
$$


(8) Generalized Lucas numbers $L(k, n)$ (Włoch [16]):

$L(k, n)=L(k, n-1)+L(k, n-k), \quad k \geq 2, n \geq 2 k$,

with $L(k, n)=n+1$ for $n=0,1, \ldots, 2 k-1$.

(9) Distance Pell numbers $\operatorname{Pd}(k, n)$ (Szynal-Liana and Włoch [5]):

$$
\begin{aligned}
\operatorname{Pd}(k, n)= & \operatorname{Pd}(k, n-1)+\operatorname{Pd}(k, n-2) \\
& +\operatorname{Pd}(k, n-k), \quad k \geq 1, n \geq k,
\end{aligned}
$$

with $\operatorname{Pd}(k, 0)=0$ and $\operatorname{Pd}(k, i)=1$ for $k \leq i+2, i \geq 1$, and $\operatorname{Pd}(k, i)=0$ for $k>i+2, i \geq 1$.

(10) Distance companion Pell numbers $Q d(k, n)$ (SzynalLiana et al. [6]):

$$
\begin{aligned}
Q d(k, n)= & Q d(k, n-1)+Q d(k, n-2) \\
& +Q d(k, n-k), \quad k \geq 1, n \geq k,
\end{aligned}
$$

with $Q d(k, 0)=k, Q d(1,1)=1$, and $Q d(k, n)=L_{n}$ for $n=1, \ldots, k-1$.

(11) Distance Jacobsthal numbers $J(k, t, n)$ (Szynal-Liana et al. [6]):

$J(k, t, n)=J(k, t, n-1)+t J(k, t, n-k)$,

$$
k \geq 2, n \geq k+1,
$$

with $J(k, t, 0)=0$ and $J(k, t, n)=1$ for $n=1, \ldots, k$.

(12) Distance Jacobsthal-Lucas numbers $\mathrm{JL}(k, t, n)$ (Szynal-Liana et al. [6]):

$\mathrm{JL}(k, t, n)=\mathrm{JL}(k, t, n-1)+t \mathrm{JL}(k, t, n-k)$,

$$
k \geq 2, n \geq k \text {, }
$$

with $\operatorname{JL}(k, t, 0)=k$ and $\operatorname{JL}(k, t, n)=1$ for $n=1, \ldots, k-$ 1 .

(13) $(2, k)$-distance Fibonacci numbers $F_{2}^{(2)}(k, n)$ of the second kind (Bednarz et al. [17]):

$$
\begin{aligned}
F_{2}^{(2)}(k, n)=F_{2}^{(2)}(k, n-2)+F_{2}^{(2)}(k, n-k), & \\
& k \geq 1, n \geq k+1,
\end{aligned}
$$

with $F_{2}^{(2)}(k, n)=0$ if $n$ is odd and $n \leq k-1, F_{2}^{(2)}(k, n)=$ 1 if $n$ is even and $n \leq k-1, F_{2}^{(2)}(k, k)=0$ if $k=1$, $F_{2}^{(2)}(k, k)=1$ if $k$ is odd and $k \geq 3$, and $F_{2}^{(2)}(k, k)=2$ if $k$ is even.

(14) $(2, k)$-distance Lucas numbers $L_{2}^{(2)}(k, n)$ of the second kind (Bednarz et al. [17]):

$$
\begin{aligned}
L_{2}^{(2)}(k, n)=L_{2}^{(2)}(k, n-2)+L_{2}^{(2)}(k, n-k), & \\
& \quad k \geq 1, n \geq k+2,
\end{aligned}
$$

with $L_{2}^{(2)}(1,0)=2$ and $L_{2}^{(2)}(k, 0)=k$ for $k \geq 2$, $L_{2}^{(2)}(k, 1)=0, L_{2}^{(2)}(1,2)=3$, and $L_{2}^{(2)}(k, k)=k$ if $k$ is odd and $L_{2}^{(2)}(k, k)=k+2$ if $k$ is even, $L_{2}^{(2)}(k, k+1)=2$ if $k$ is odd and $k \neq 1, L_{2}^{(2)}(k, k+1)=0$ if $k$ is even, $L_{2}^{(2)}(k, n)=0$ if $n$ is odd and $3 \leq n \leq k-1$, and $L_{2}^{(2)}(k, n)=2$ if $n$ is even and $2 \leq n \leq k-1$.

For most of these numbers also some graph interpretations with respect to distance independent sets or matchings were studied (see, e.g., $[5,6,8,10,12,18-20]$ ).

\section{Main Results}

The main purpose of this section is to give a total graph interpretation for numbers of the Fibonacci type with respect to a special edge colouring of some graphs.

Let $G$ be an undirected, connected, simple graph. Let $\mathscr{I}=$ $\{1, \ldots, k\}, k \geq 2$, and let $\mathscr{I}_{i}=\left\{1, \ldots, b_{i}\right\}, b_{i} \geq 1$. In particular, $\mathscr{I}_{i}$ can be empty (then we put $b_{i}=0$ ). Let $\mathscr{C}=\bigcup_{i \in \mathscr{I}} \mathscr{C}_{i}$ be a nonempty family of colours, where $\mathscr{C}_{i}=\left\{i A_{j} ; j \in \mathscr{I}_{i}\right\}$ for $i=1, \ldots, k$. The set $\mathscr{C}_{i}$ will be called the set of $b_{i}$ shades of the colour $i$. Consequently, for all $i \neq p, 1 \leq i, p \leq k$, $i A_{j} \neq p A_{j}$ holds and this implies that the family $\mathscr{C}$ has exactly $\sum_{i=1}^{k}\left|\mathscr{C}_{i}\right|=\sum_{i=1}^{k} b_{i}$ colours.

A graph $G$ is $\left(i A_{j} ; i \in \mathscr{I}, j \in \mathscr{I}_{i}\right)$-edge coloured by monochromatic paths if for every maximal $i A_{j}$-monochromatic subgraph $H$ of $G$, where $i A_{j} \in \mathscr{C}_{i}, 1 \leq i \leq k$, $1 \leq j \leq b_{i}$, there is a partition of $H$ into edge disjoint paths of the length $i$. Clearly, if $b_{1} \neq 0$ then $\left(i A_{j} ; i \in \mathscr{I}, j \in \mathscr{I}_{i}\right)$-edge colouring by monochromatic paths always exists. With this type of edge colouring of a graph, we associate the following graph parameter.

Let $G$ be a graph which can be $\left(i A_{j} ; i \in \mathscr{I}, j \in \mathscr{I}_{i}\right)$ edge coloured by monochromatic paths. Let $\mathscr{F}$ be a family of distinct $\left(i A_{j} ; i \in \mathscr{I}, j \in \mathscr{I}_{i}\right.$ )-edge coloured graphs obtained by colouring of a graph $G$ and

$$
\mathscr{F}=\left\{G^{(1)}, G^{(2)}, \ldots, G^{(l)}\right\}, \quad l \geq 1,
$$

where $G^{(p)}, 1 \leq p \leq l$, denotes a graph obtained by $\left(i A_{j}\right.$; $i \in \mathscr{I}, j \in \mathscr{I}_{i}$ )-edge colouring by monochromatic paths of a graph $G$.

For $\left(i A_{j} ; i \in \mathscr{I}, j \in \mathscr{I}_{i}\right)$-edge coloured graph $G^{(p)}$ by $\theta\left(G^{(p)}\right)$, we denote the number of all partitions of $G^{(p)}$ for $1 \leq$ $p \leq l$. Let

$$
\sigma_{\left(i A_{j} ; i \in \mathscr{F}_{\left., j \in \mathcal{F}_{i}\right)}\right.}(G)=\sum_{p=1}^{l} \theta\left(G^{(p)}\right)
$$

Considering the $k$ th-order linear recurrence relation (1), we will show that there is a connection between this

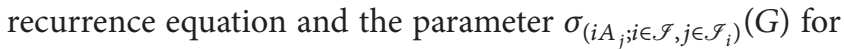
a special graph $G$. 
Theorem 4. Let $k \geq 2$ and $m \geq k$ be integers. Then,

$$
\begin{aligned}
& \sigma_{\left(i A_{j} ; i \in \mathcal{I}_{\left., j \in \mathcal{F}_{i}\right)}(\mathbb{P}(m))\right.} \\
& =b_{1} \sigma_{\left(i A_{j} ; i \in \mathcal{F}_{\left., j \in \mathcal{F}_{i}\right)}\right.}(\mathbb{P}(m-1))+\cdots \\
& \quad+b_{k} \sigma_{\left(i A_{j} ; i \in \mathcal{I}_{\left., j \in \mathcal{F}_{i}\right)}\right.}(\mathbb{P}(m-k)) .
\end{aligned}
$$

Proof. Assume that $m \geq k$ and let us consider $\left(i A_{j} ; i \in \mathscr{I}\right.$, $j \in \mathscr{I}_{i}$ )-edge colouring by monochromatic paths of the graph $\mathbb{P}(m)$ with the numbering of its edges in the natural fashion.

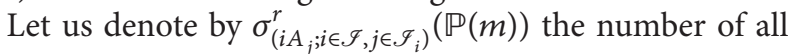
$\left(i A_{j} ; i \in \mathscr{I}, j \in \mathscr{I}_{i}\right.$ )-edge colourings by monochromatic paths of the graph $\mathbb{P}(m)$, such that the last edge of $\mathbb{P}(m)$ is coloured by the colour from the fixed set $\mathscr{C}_{r}$, where $1 \leq r \leq k$. It is clear that

$$
\sigma_{\left(i A_{j} ; i \in \mathscr{F}_{\left., j \in \mathcal{F}_{i}\right)}\right.}(\mathbb{P}(m))=\sum_{r=1}^{k} \sigma_{\left(i A_{j} ; i \in \mathcal{F}_{\left., j \in \mathscr{I}_{i}\right)}\right.}^{r}(\mathbb{P}(m)) .
$$

If the edge $e_{m} \in \mathbb{P}(m)$ is coloured by one of the shades of colour $r$, that is, by $r A_{j}, 1 \leq r \leq k, 1 \leq j \leq b_{r}$, then, according to the definition of $\left(i A_{j} ; i \in \mathscr{I}, j \in \mathscr{I}_{i}\right)$-edge colouring by monochromatic paths, the number $\sigma_{\left(i A_{j} ; i \in \mathscr{I}_{\left., j \in \mathcal{F}_{i}\right)}^{r}\right.}(\mathbb{P}(m))=$ $\sigma_{\left(i A_{j} ; i \in \mathscr{I}_{\left., j \in \mathscr{I}_{i}\right)}\right.}(\mathbb{P}(m-r))$ is the number of all $\left(i A_{j} ; i \in \mathscr{I}\right.$, $j \in \mathscr{I}_{i}$ )-edge colourings by monochromatic paths of the graph $\mathbb{P}(m-r)$. Taking into account that we consider $b_{r}$ shades of every colour $r$, we get

$$
\begin{aligned}
& \sigma_{\left(i A_{j} ; i \in \mathcal{F}, j \in \mathcal{F}_{i}\right)}(\mathbb{P}(m)) \\
& =b_{1} \sigma_{\left(i A_{j} ; i \in \mathcal{F}, j \in \mathcal{I}_{i}\right)}(\mathbb{P}(m-1))+\cdots \\
& \quad+b_{k} \sigma_{\left(i A_{j} ; i \in \mathcal{F}_{\left., j \in \mathcal{F}_{i}\right)}\right.}(\mathbb{P}(m-k)),
\end{aligned}
$$

which ends the proof.

For the special case of $\sigma_{\left(i A_{j} ; i \in \mathcal{I}_{\left., j \in \mathcal{F}_{i}\right)}\right.}(\mathbb{P}(m))$, we can prove the following.

Theorem 5. Let $k$ and $m$ be integers. Then,

(1) $\sigma_{\left(A_{1}, 2 A_{1}\right)}(\mathbb{P}(m))=F_{m}$ for $m \geq 1$,

(2) $\sigma_{\left(A_{1}, A_{2}, 2 A_{1}\right)}(\mathbb{P}(m))=P_{m+1}$ for $m \geq 1$,

(3) $\sigma_{\left(A_{1}, 2 A_{1}, 2 A_{2}\right)}(\mathbb{P}(m))=J_{m+1}$ for $m \geq 1$,

(4) $\sigma_{\left(A_{1}, 2 A_{1}, 3 A_{1}\right)}(\mathbb{P}(m))=T_{m+1}^{*}$ for $m \geq 1$,

(5) $\sigma_{\left(2 A_{1}, 3 A_{1}\right)}(\mathbb{P}(m))=P v(m-2)$ for $m \geq 2$,

(6) $\sigma_{\left(A_{1}, 2 A_{1}, \ldots, k A_{1}\right)}(\mathbb{P}(m))=f_{m+k-1}$ for $k \geq 2$ and $m \geq k-1$,

(7) $\sigma_{\left(A_{1}, k A_{1}\right)}(\mathbb{P}(m))=F(k, m-k+1)$ for $k \geq 2$ and $m \geq$ $k-1$,

(8) $\sigma_{\left(A_{1}, \ldots, A_{k}, 2 A_{1}\right)}(\mathbb{P}(m))=F_{k}(m+1)$ for $k \geq 1$ and $m \geq 1$,

(9) $\sigma_{\left(A_{1},(k-1) A_{1}, k A_{1}\right)}(\mathbb{P}(m))=P(k, m-k+3)$ for $k \geq 2$ and $m \geq k-3$,

(10) $\sigma_{\left(A_{1}, A_{2}, 2 A_{1}, \ldots, 2 A_{k}\right)}(\mathbb{P}(m))=P_{k}(m+1)$ for $k \geq 1$ and $m \geq 1$,
(11) $\sigma_{\left(A_{1}, 2 A_{1}, k A_{1}\right)}(\mathbb{P}(m))=\operatorname{Pd}(k, m+k-2)$ for $k \geq 1$ and $m \geq k-2$,
(12) $\sigma_{\left(A_{1}, k A_{1}, k A_{2}, \ldots, k A_{t}\right)}(\mathbb{P}(m))=J(k, t, m+1)$ for $k \geq 2$ and $m \geq 1$,
(13) $\sigma_{\left(2 A_{1}, k A_{1}\right)}(\mathbb{P}(m))=F_{2}^{(2)}(k, m)$ for $k \geq 1$ and $m \geq 1$.

Proof. Let $m$ be as in each statement of the theorem. For the initial terms in each case, we determine the number $\sigma_{\left(i A_{j} ; i \in \mathscr{F}_{\left., j \in \mathscr{F}_{i}\right)}\right.}(G)$ by inspection. We will analyze some cases.

(1) Consider $\left(A_{1}, 2 A_{1}\right)$-edge colouring by monochromatic paths of $\mathbb{P}(m)$. If $m=1$ then the unique edge of $\mathbb{P}(1)$ is coloured by colour $A_{1}$. If $m=2$ then we have a path with two edges. So there are exactly two $\left(A_{1}, 2 A_{1}\right)$-edge colourings of $\mathbb{P}(2)$ in the first case using colour $A_{1}$ and in the second case using the colour $2 A_{1}$. In each case, the graph $\mathbb{P}(2)$ is monochromatic. Then, $\sigma_{\left(A_{1}, 2 A_{1}\right)}(\mathbb{P}(1))=1=F_{1}$ and $\sigma_{\left(A_{1}, 2 A_{1}\right)}(\mathbb{P}(2))=2=F_{2}$.

(2) Now consider $\left(A_{1}, A_{2}, 2 A_{1}\right)$-edge colouring by monochromatic paths of $\mathbb{P}(m)$. If $m=1$ then there are exactly two $\left(A_{1}, A_{2}, 2 A_{1}\right)$-edge colourings of $\mathbb{P}(1)$. The unique edge can be coloured either by $A_{1}$ or by $A_{2}$. Thus, $\sigma_{\left(A_{1}, A_{2}, 2 A_{1}\right)}(\mathbb{P}(1))=2=P_{2}$. Let $m=2$. Then, using only colours $A_{1}$ and $A_{2}$ we can colour edges of the graph $\mathbb{P}(2)$ as follows: $A_{1} A_{1}, A_{1} A_{2}, A_{2} A_{1}, A_{2} A_{2}$. Moreover, there is the unique colouring of the graph $\mathbb{P}(2)$ using the colour $2 A_{1}$. Then, $\mathbb{P}(2)$ is $2 A_{1}$-monochromatic. Consequently, $\sigma_{\left(A_{1}, A_{2}, 2 A_{1}\right)}(\mathbb{P}(2))=5=P_{3}$.

(7) Consider $\left(A_{1}, k A_{1}\right)$-edge colouring by monochromatic paths of $\mathbb{P}(m)$. If $m=k-1$, then there is a unique $\left(A_{1}, k A_{1}\right)$-edge colouring using only colour $A_{1}$. Let $m=k+i$, where $i=0, \ldots, k-1$. Then, in the path $\mathbb{P}(m)$, one $k A_{1}$ monochromatic subpath can exist at most or $\mathbb{P}(m)$ is $A_{1}$ monochromatic. Because $k A_{1}$-monochromatic path can be chosen on $i+1$ ways, $\sigma_{\left(A_{1}, k A_{1}\right)}(\mathbb{P}(m))=i+2=F(k, i+1)$.

In the same way, we can verify the initial conditions in the remaining cases (3)-(6) and (8)-(13).

By the initial conditions and by Theorem 4 the result follows.

Analogously as for paths $\mathbb{P}(m)$ we can prove the following theorem for the cycle $\mathbb{C}(m)$.

Theorem 6. Let $k \geq 2$ and $m \geq k-1$ be integers. Then,

$$
\begin{aligned}
\sigma_{\left(i A_{j} ; i \in \mathscr{J}_{\left., j \in \mathscr{I}_{i}\right)}\right.}(\mathbb{C}(m)) \\
=\sum_{r=1}^{k} b_{r} r \sigma_{\left(i A_{j} ; i \in \mathscr{I}_{\left., j \in \mathscr{I}_{i}\right)}\right.}(\mathbb{P}(m-r)) .
\end{aligned}
$$

Proof. Let $\mathbb{C}(m)$ be a cycle of a size $m$ with the numbering

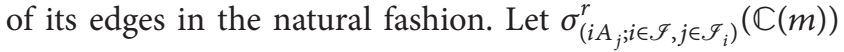
denote the number of all $\left(i A_{j} ; i \in \mathscr{I}, j \in \mathscr{I}_{i}\right)$-edge colourings by monochromatic paths of $\mathbb{C}(m)$ such that the edge $e_{1} \in$ $E(\mathbb{C}(m))$ has the colour from the fixed $\mathscr{C}_{r}$, where $1 \leq r \leq k$. Clearly,

$$
\sigma_{\left(i A_{j} ; i \in \mathcal{F}, j \in \mathscr{F}_{i}\right)}(\mathbb{C}(m))=\sum_{r=1}^{k} \sigma_{\left(i A_{j} ; i \in \mathcal{F}_{\left., j \in \mathcal{F}_{i}\right)}^{r}\right.}(\mathbb{C}(m)) .
$$


If the edge $e_{1} \in E(\mathbb{C}(m))$ is coloured by the colour $r A_{j}$, where $1 \leq r \leq k$ and $1 \leq j \leq b_{r}$, then

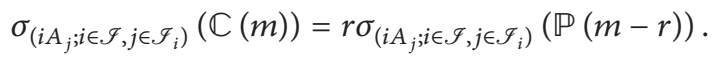

The factor $r$ follows from the fact that the edge $e_{1}$ can belong to an $r A_{j}$-monochromatic path (of length $r$ ) on $r$ ways, $1 \leq$ $r \leq i$.

Moreover, in the set $\mathscr{C}_{r}$, we have $b_{r}$ shades of the colour $r$ and $r$ can be chosen on $k$ ways; thus,

$$
\begin{aligned}
\sigma_{\left(i A_{j} ; i \in \mathscr{I}, j \in \mathscr{I}_{i}\right)}(\mathbb{C}(m)) \\
=\sum_{r=1}^{k} b_{r} r \sigma_{\left(i A_{j} ; i \in \mathcal{F}_{\left., j \in \mathscr{F}_{i}\right)}\right.}(\mathbb{P}(m-r)),
\end{aligned}
$$

which ends the proof.

Using the above theorem, we can give a graph interpretation for the cyclic version of the Fibonacci type. Firstly, we recall some identities given in $[6,13,16,17]$ which will be useful to prove the next theorem.

For $n \geq 1$ and $k \geq 1$,

$$
L_{k}(n)=F_{k}(n-1)+F_{k}(n+1)
$$

(see [13]). For $k \geq 2$ and $n \geq 2 k$,

$$
L(k, n)=k F(k,(n-2 k+1))+F(k, n-k)
$$

(see [16]). For $k \geq 3$ and $n \geq k$,

$$
\begin{aligned}
\mathrm{Qd}(k, n)= & \operatorname{Pd}(k, n+k-3)+2 \operatorname{Pd}(k, n+k-4) \\
& +k \operatorname{Pd}(k, n-2)
\end{aligned}
$$

(see [6]). For $k \geq 3, n \geq k-1$, and $t \geq 1$,

$$
\mathrm{JL}(k, t, n)=J(k, t, n+1)+t(k-1) J(k, t, n-k+1)
$$

(see [6]). For $k \geq 2$ and $n \geq k$,

$$
L_{2}^{(2)}(k, n)=2 F_{2}^{(2)}(k, n-2)+k F_{2}^{(2)}(k, n-k)
$$

(see [17]).

To prove the next theorem, we need the following lemma.

Lemma 7. Let $k \geq 1$ and $m \geq 1$ be integers. Then,

$$
2 P_{k}(m)+2 k P_{k}(m-1)=Q_{k}(m) .
$$

Proof (by induction on $m$ ). If $m=1$, then $2 P_{k}(1)+2 k P_{k}(0)=$ $2=Q_{k}(1)$. Assume that formula (40) holds for an arbitrary $m$. We will prove it for $m+1$. By the recurrence definitions of the numbers $P_{k}(m)$ and $Q_{k}(m)$ and by induction hypothesis, we have

$$
\begin{aligned}
Q_{k}(m+1)= & 2 Q_{k}(m)+k Q_{k}(m-1) \\
= & 2\left(2 P_{k}(m)+2 k P_{k}(m-1)\right) \\
& +k\left(2 P_{k}(m-1)+2 k P_{k}(m-2)\right) \\
= & 2\left(2 P_{k}(m)+k P_{k}(m-1)\right) \\
& +2 k\left(2 P_{k}(m-1)+k P_{k}(m-2)\right) \\
= & 2 P_{k}(m+1)+2 k P_{k}(m),
\end{aligned}
$$

which ends the proof of Lemma 7.
Theorem 8. Let $k$ and $m$ be integers. Then,

(1) $\sigma_{\left(A_{1}, 2 A_{1}\right)}(\mathbb{C}(m))=L_{m}$, for $m \geq 3$,

(2) $\sigma_{\left(A_{1}, 2 A_{1}, 2 A_{2}\right)}(\mathbb{C}(m))=j_{m}$, for $m \geq 3$,

(3) $\sigma_{\left(2 A_{1}, 3 A_{1}\right)}(\mathbb{C}(m))=\operatorname{Pr}(m)$, for $m \geq 5$,

(4) $\sigma_{\left(A_{1}, \ldots, A_{k}, 2 A_{1}\right)}(\mathbb{C}(m))=L_{k}(m)$, for $k \geq 1$ and $m \geq 3$,

(5) $\sigma_{\left(A_{1}, A_{2}, 2 A_{1}, \ldots, 2 A_{k}\right)}(\mathbb{C}(m))=Q_{k}(m)$, for $k \geq 1$ and $m \geq$ 3 ,

(6) $\sigma_{\left(A_{1}, k A_{1}\right)}(\mathbb{C}(m))=L(k, m)$, for $k \geq 2$ and $m \geq 2 k-1$,

(7) $\sigma_{\left(A_{1}, 2 A_{1}, k A_{1}\right)}(\mathbb{C}(m))=Q d(k, m)$, for $k \geq 3$ and $m \geq k$,

(8) $\sigma_{\left(A_{1}, k A_{1}, \ldots, k A_{t}\right)}(\mathbb{C}(m))=J L(k, t, m)$, for $k \geq 3$ and $m \geq$ $k+1$,

(9) $\sigma_{\left(2 A_{1}, k A_{1}\right)}(\mathbb{C}(m))=L_{2}^{(2)}(k, m)$, for $k \geq 2$ and $m \geq k+1$.

Proof. Let $k$ and $m$ be as in each statement of the theorem.

(1) From Theorem 6, we have that

$$
\begin{aligned}
\sigma_{\left(A_{1}, 2 A_{1}\right)}(\mathbb{C}(m))= & \sigma_{\left(A_{1}, 2 A_{1}\right)}(\mathbb{P}(m-1)) \\
& +2 \sigma_{\left(A_{1}, 2 A_{1}\right)}(\mathbb{P}(m-2)) .
\end{aligned}
$$

Moreover, by Theorem 5, by the recurrence relation of Fibonacci numbers and the well-known identity for Lucas numbers follows

$$
\sigma_{\left(A_{1}, 2 A_{1}\right)}(\mathbb{C}(m))=F_{m-1}+2 F_{m-2}=F_{m}+F_{m-2}=L_{m},
$$

which ends the proof of (1).

(2) From Theorems 5 and 6, by the recurrence relation of Jacobsthal numbers and the well-known identity for Jacobsthal numbers, we obtain

$$
\begin{aligned}
\sigma_{\left(A_{1}, 2 A_{1}, 2 A_{2}\right)}(\mathbb{C}(m))= & \sigma_{\left(A_{1}, 2 A_{1}, 2 A_{2}\right)}(\mathbb{P}(m-1)) \\
& +4 \sigma_{\left(A_{1}, 2 A_{1}, 2 A_{2}\right)}(\mathbb{P}(m-2)) \\
= & J_{m}+4 J_{m-1}=J_{m+1}+2 J_{m-1} \\
= & j_{m}
\end{aligned}
$$

which ends the proof of (2).

Analogously, like in the previous cases, using Theorems 5 and 6 and Lemma 7 and applying known identities for considered numbers, we prove the other conditions such as in what follows.

(3) $\sigma_{\left(2 A_{1}, 3 A_{1}\right)}(\mathbb{C}(m))=2 \sigma_{\left(2 A_{1}, 3 A_{1}\right)}(\mathbb{P}(m-2))+$ $3 \sigma_{\left(2 A_{1}, 3 A_{1}\right)}(\mathbb{P}(m-3))=2 \operatorname{Pv}(m-4)+3 \operatorname{Pv}(m-5)=2 \operatorname{Pv}(m-$ 2) $+\operatorname{Pv}(m-5)=\operatorname{Pr}(m)$.

(4) $\sigma_{\left(A_{1}, \ldots, A_{k}, 2 A_{1}\right)}(\mathbb{C}(m))=k \sigma_{\left(A_{1}, \ldots, A_{k}, 2 A_{1}\right)}(\mathbb{P}(m-1))+$ $2 \sigma_{\left(A_{1}, \ldots, A_{k}, 2 A_{1}\right)}(\mathbb{P}(m-2))=k F_{k}(m)+2 F_{k}(m-1)=k F_{k}(m)+$ $F_{k}(m-1)+F_{k}(m-1)=F_{k}(m+1)+F_{k}(m-1)=L_{k}(m)$ by (35).

(5) $\sigma_{\left(A_{1}, A_{2}, 2 A_{1}, \ldots, 2 A_{k}\right)}(\mathbb{C}(m))=2 \sigma_{\left(A_{1}, A_{2}, 2 A_{1}, \ldots, 2 A_{k}\right)}(\mathbb{P}(m-$ $1))+2 k \sigma_{\left(A_{1}, A_{2}, 2 A_{1}, \ldots, 2 A_{k}\right)}(\mathbb{P}(m-2))=2 P_{k}(m)+2 k P_{k}(m-1)=$ $Q_{k}(m)$ by Lemma 7 .

(6) $\sigma_{\left(A_{1}, k A_{1}\right)}(\mathbb{C}(m))=\sigma_{\left(A_{1}, k A_{1}\right)}(\mathbb{P}(m-1))+k \sigma_{\left(A_{1}, k A_{1}\right)}(\mathbb{P}(m$ $-k))=F(k, m-k)+k F(k, m-2 k+1)=L(k, m)$ by $(36)$, which ends the proof of (6). 
(7) $\sigma_{\left(A_{1}, 2 A_{1}, k A_{1}\right)}(\mathbb{C}(m))=\sigma_{\left(A_{1}, 2 A_{1}, k A_{1}\right)}(\mathbb{P}(m-1))+$ $2 \sigma_{\left(A_{1}, 2 A_{1}, k A_{1}\right)}(\mathbb{P}(m-2))+k \sigma_{\left(A_{1}, 2 A_{1}, k A_{1}\right)}(\mathbb{P}(m-k))=\operatorname{Pd}(k, m+$ $k-3)+2 \operatorname{Pd}(k, m+k-4)+k \operatorname{Pd}(k, m-2)=Q d(k, m)$ by $(37)$, which ends the proof of (7).

(8) $\sigma_{\left(A_{1}, k A_{1}, \ldots, k A_{t}\right)}(\mathbb{C}(m))=\sigma_{\left(A_{1}, k A_{1}, \ldots, k A_{t}\right)}(\mathbb{P}(m-1))+$ $t k \sigma_{\left(A_{1}, k A_{1}, \ldots, k A_{t}\right)}(\mathbb{P}(m-k))=J(k, t, m)+t k J(k, t, m-k+1)=$ $J(k, t, m)+t(k-1) J(k, t, m-k+1)+t J(k, t, m-k+1)=$ $J(k, t, m+1)+t(k-1) J(k, t, m-k+1)=\mathrm{JL}(k, t, m)$ by $(38)$, which ends the proof of (8).

(9) $\sigma_{\left(2 A_{1}, k A_{1}\right)}(\mathbb{C}(m))=2 \sigma_{\left(2 A_{1}, k A_{1}\right)}(\mathbb{P}(m-2))+$ $k \sigma_{\left(2 A_{1}, k A_{1}\right)}(\mathbb{P}(m-k))=2 F_{2}^{(2)}(k, m-2)+k F_{2}^{(2)}(k, m-k)=$ $L_{2}^{(2)}(k, m)$ by (39), which ends the proof of (9).

Thus, the theorem is proved.

\section{3. $\left(A_{1}, 2 A_{1}\right)$-Edge Colouring in Trees}

We can study $\left(i A_{j} ; i \in \mathscr{I}, j \in \mathscr{I}_{i}\right)$-edge colouring by monochromatic paths in distinct classes of graphs. For arbitrary $k$ and $b_{i}, i \geq 0$, the problem seems to be difficult but some interesting results can be obtained for fixed $k$ and $b_{i}, i=1, \ldots, k$. Assume that $k=2$ and $b_{1}=b_{2}=1$. Then, the $\left(A_{1}, 2 A_{1}\right)$-edge colouring always exists in an arbitrary graph $G$. In this section, we consider the number of all $\left(A_{1}, 2 A_{1}\right)$ edge colourings in trees.

Theorem 9. Let $\mathbb{T}(m)$ be a tree of size $m, m \geq 1$. Then,

$$
\begin{aligned}
F_{m} & \leq \sigma_{\left(A_{1}, 2 A_{1}\right)}(\mathbb{T}(m)) \\
& \leq 1+\sum_{j \geq 1}\left(\begin{array}{l}
m \\
2 j
\end{array}\right) \prod_{p=0}^{j-1}[2 j-(2 p+1)] .
\end{aligned}
$$

Moreover, for

$$
\sigma_{\left(A_{1}, 2 A_{1}\right)}(\mathbb{P}(m))=F_{m}, \quad \mathbb{T}(m)=\mathbb{P}(m)
$$

and for

$$
\begin{array}{r}
\sigma_{\left(A_{1}, 2 A_{1}\right)}(\mathbb{S}(m))=1+\sum_{j \geq 1}\left(\begin{array}{l}
m \\
2 j
\end{array}\right) \prod_{p=0}^{j-1}[2 j-(2 p+1)], \\
\mathbb{T}(m)=\mathbb{S}(m) .
\end{array}
$$

Proof. Let $\mathbb{T}(m)$ be a tree of size $m, m \geq 1$. Firstly, we will show that

$$
\sigma_{\left(A_{1}, 2 A_{1}\right)}(\mathbb{T}(m)) \geq F_{m} .
$$

We prove it by induction on $m$. If $m=1,2$ then $\mathbb{T}(m)$ is isomorphic to $\mathbb{P}(m)$ and the result is obvious. Let $x \in$ $V(\mathbb{T}(m))$ be a leaf incident with the edge $x y \in E(\mathbb{T}(m))$ such that $y z \in E(\mathbb{T}(m))$ and $\mathbb{T}(m) \backslash\{x y, y z\}$ is isomorphic to either $\mathbb{V}(m-2) \cup\{x, z\}$ or $\mathbb{T}(m-2) \cup\{x, y\}$. Such leaf $x \in V(\mathbb{T}(m))$ always exists in a tree, by the basic tree properties. This means that $\mathbb{T}(m-2)$ is a tree. Since isolated vertices are not taken into consideration in $\left(A_{1}, 2 A_{1}\right)$-edge colourings, it suffices to consider the tree $\mathbb{T}(m-2)$.
Assume that $x, y$, and $z$ are as above and consider the following possibilities.

(i) $x y \in E(\mathbb{T}(m))$ has the colour $A_{1}$.

Then, the subgraph $\mathbb{T}(m) \backslash\{x y\}$ is isomorphic to $\mathbb{T}(m-1) \cup$ $\{x\}$ and has the $\left(A_{1}, 2 A_{1}\right)$-edge colouring. By our assumption, there is at least $F_{m-1} \operatorname{such}\left(A_{1}, 2 A_{1}\right)$-edge colourings of $\mathbb{T}(m)$ in this case.

(ii) $x y \in E(\mathbb{T}(m))$ has the colour $2 A_{1}$.

Then, by the definition of the $\left(A_{1}, 2 A_{1}\right)$-edge colouring, it immediately follows that there is a $2 A_{1}$-monochromatic subgraph with a partition $\theta$ into $2 A_{1}$-monochromatic paths of length 2 .

Let $H \leq \mathbb{T}(m)$ be a maximal $2 A_{1}$-monochromatic subgraph with a partition $\theta$. Let $y z \in E(H)$ have the colour $2 A_{1}$ and the path $x y, y z$ belongs to $\theta$. Then, the subgraph $\mathbb{T}(m) \backslash\{x y, y z\}$ also has $\left(A_{1}, 2 A_{1}\right)$-edge colouring and $|E(\mathbb{T}(m) \backslash\{x y, y z\})|=m-2$. Clearly, the edge $x y \in$ $E(\mathbb{T}(m))$ belongs to at least one partition $\theta$ of the $2 A_{1}$ monochromatic subgraph of $\mathbb{T}(m)$ into $2 A_{1}$-monochromatic paths of length 2. Consequently, if $x y \in E(\mathbb{T}(m))$ has the colour $2 A_{1}$, then the number of all $\left(A_{1}, 2 A_{1}\right)$-edge colourings of $\mathbb{}(m)$ is greater than or equal to the number $\sigma_{\left(A_{1}, 2 A_{1}\right)}(\mathbb{T}(m) \backslash$ $\{x y, y z\})$. Since $\mathbb{T}(m) \backslash\{x y, y z\}$ has two isolated vertices, it suffices to consider $\mathbb{T}(m-2)$, so $\sigma_{\left(A_{1}, 2 A_{1}\right)}(\mathbb{T}(m) \backslash\{x y, y z\}) \geq$ $\sigma_{\left(A_{1}, 2 A_{1}\right)}(\mathbb{T}(m-2)) \geq F_{m-2}$ by the induction hypothesis. From the above, $\sigma_{\left(A_{1}, 2 A_{1}\right)}(\mathbb{T}(m)) \geq F_{m-1}+F_{m-2}=F_{m}$. By Theorem 5, the equality is obvious.

Now we show that

$$
\sigma_{\left(A_{1}, 2 A_{1}\right)}(\mathbb{T}(m)) \leq 1+\sum_{j \geq 1}\left(\begin{array}{l}
m \\
2 j
\end{array}\right) \prod_{p=0}^{j-1}[2 j-(2 p+1)] .
$$

Since $\mathbb{T}(m)$ is a connected graph, the maximum value is attained if both edges are adjacent. This means that $\mathbb{T}(m)$ is the star. Then, there is a maximum number of substars with even number of edges. Consequently, it suffices to calculate the number of all substars of a star with even number of edges. Since $|E(\mathbb{T}(m))|=m$ and we choose even number of edges, we have at least $\sum_{j \geq 1}\left(\begin{array}{c}m \\ 2 j\end{array}\right)$ possibilities of choosing of subset of even edges. Moreover, $2 j$ edges can be partitioned into $(2 j-1)(2 j-3) \cdots \cdot 3 \cdot 1$ ways. Additionally, $\mathbb{T}(m)$ can be $A_{1}$-monochromatic.

All this together gives that $\sigma_{\left(A_{1}, 2 A_{1}\right)}(\mathbb{T}(m)) \leq 1+$ $\sum_{j \geq 1}\left(\begin{array}{c}m \\ 2 j\end{array}\right) \prod_{p=0}^{j-1}[2 j-(2 p+1)]$. The proof of equalities is obvious.

\section{Concluding Remarks}

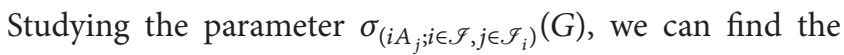
graph interpretation with respect to $\left(i A_{j} ; i \in \mathscr{I}, j \in \mathscr{I}_{i}\right)$ edge colourings by monochromatic paths for other numbers of the Fibonacci type not considered in this paper. For some of them, such as the Tribonacci numbers $T_{n}$, it does not work in paths and cycles. We can ask about the existence of a graph $G$ for which $\sigma_{\left(i A_{j} ; i \in \mathcal{F}_{\left., j \in \mathscr{F}_{i}\right)}\right.}(G)=T_{n}$. It is also interesting to consider problems of determining the parameter $\sigma_{\left(i A_{j} ; i \in \mathcal{F}, j \in \mathscr{F}_{i}\right)}(G)$ in distinct classes, clearly for a special $\left(i A_{j}\right.$; $i \in \mathscr{I}, j \in \mathscr{I}_{i}$ )-edge colouring by monochromatic paths. 


\section{Conflict of Interests}

The authors declare that there is no conflict of interests regarding the publication of this paper.

\section{Acknowledgment}

The authors wish to thank the referee for all suggestions which improved this paper.

\section{References}

[1] C. Berge, Principles of Combinatorics, Academic Press, New York, NY, USA, 1971.

[2] R. Diestel, Graph Theory, Springer, New York, NY, USA, 2005.

[3] The On-Line Encyclopedia of Integer Sequences, https://oeis .org/.

[4] H. Prodinger and R. F. Tichy, "Fibonacci numbers of graphs," The Fibonacci Quarterly, vol. 20, no. 1, pp. 16-21, 1982.

[5] A. Szynal-Liana and I. Włoch, "On distance Pell numbers and their connections with Fibonacci numbers," Ars Combinatoria A, vol. 113, pp. 65-75, 2014.

[6] A. Szynal-Liana, A. Włoch, and I. Włoch, "On generalized Pell numbers generated by Fibonacci and Lucas numbers," Ars Combinatoria, vol. 115, pp. 411-423, 2014.

[7] S. Wagner and I. Gutman, "Maxima and minima of the Hosoya index and the Merrifield-Simmons index. A survey of results and techniques," Acta Applicandae Mathematicae, vol. 112, no. 3, pp. 323-346, 2010.

[8] I. Włoch, "Trees with extremal numbers of maximal independent sets including the set of leaves," Discrete Mathematics, vol. 308, no. 20, pp. 4768-4772, 2008.

[9] E. P. Miles Jr., "Generalized Fibonacci numbers and associated matrices," The American Mathematical Monthly, vol. 67, no. 8, pp. 745-752, 1960.

[10] M. Kwaśnik and I. Włoch, "The total number of generalized stable sets and kernels of graphs," Ars Combinatoria, vol. 55, pp. 139-146, 2000.

[11] S. Falcón and Á. Plaza, "The $k$-Fibonacci sequence and the Pascal 2-triangle," Chaos, Solitons \& Fractals, vol. 33, no. 1, pp. 38-49, 2007.

[12] I. Włoch, "On generalized Pell numbers and their graph representations," Commentationes Mathematicae, vol. 48, no. 2 , pp. 169-175, 2008.

[13] S. Falcon, "On the $k$-lucas numbers," International Journal of Contemporary Mathematical Sciences, vol. 6, no. 21, pp. 10391050, 2011.

[14] P. Catarino, "On some identities and generating functions for $k$ Pell numbers," International Journal of Mathematical Analysis, vol. 7, no. 38, pp. 1877-1884, 2013.

[15] P. Catarino and P. Vasco, "On some identities and generating functions for k-Pell-Lucas sequence," Applied Mathematical Sciences, vol. 7, no. 98, pp. 4867-4873, 2013.

[16] A. Włoch, "Some identities for the generalized Fibonacci numbers and the generalized Lucas numbers," Applied Mathematics and Computation, vol. 219, no. 10, pp. 5564-5568, 2013.

[17] U. Bednarz, D. Bród, I. Włoch, and M. Wołowiec-Musiał, "On three types of $(2 ; \mathrm{k})$-distance Fibonacci numbers and number decompositions," Ars Combinatoria, vol. 118, pp. 391-405, 2015.
[18] U. Bednarz, D. Bród, and M. Wołowiec-Musiał, "On two types of (2; k)-distance Lucas numbers," Ars Combinatoria, vol. 115, pp. 467-479, 2014.

[19] A. Włoch, "On generalized Fibonacci numbers and $k$-distance $K_{p}$-matchings in graphs," Discrete Applied Mathematics, vol. 160, no. 9, pp. 1399-1405, 2012.

[20] I. Włoch, U. Bednarz, D. Bród, A. Włoch, and M. WołowiecMusiał, "On a new type of distance Fibonacci numbers," Discrete Applied Mathematics, vol. 161, no. 16-17, pp. 2695-2701, 2013. 


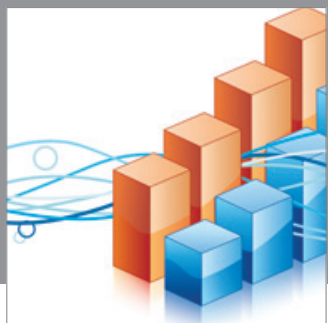

Advances in

Operations Research

mansans

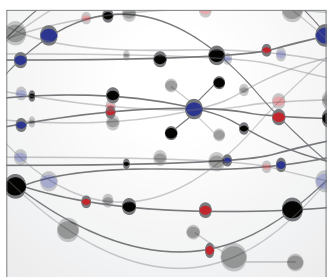

The Scientific World Journal
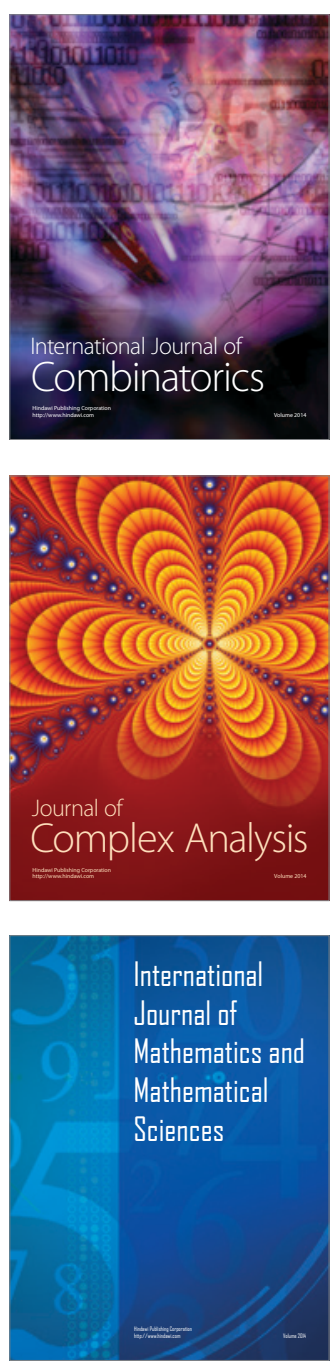
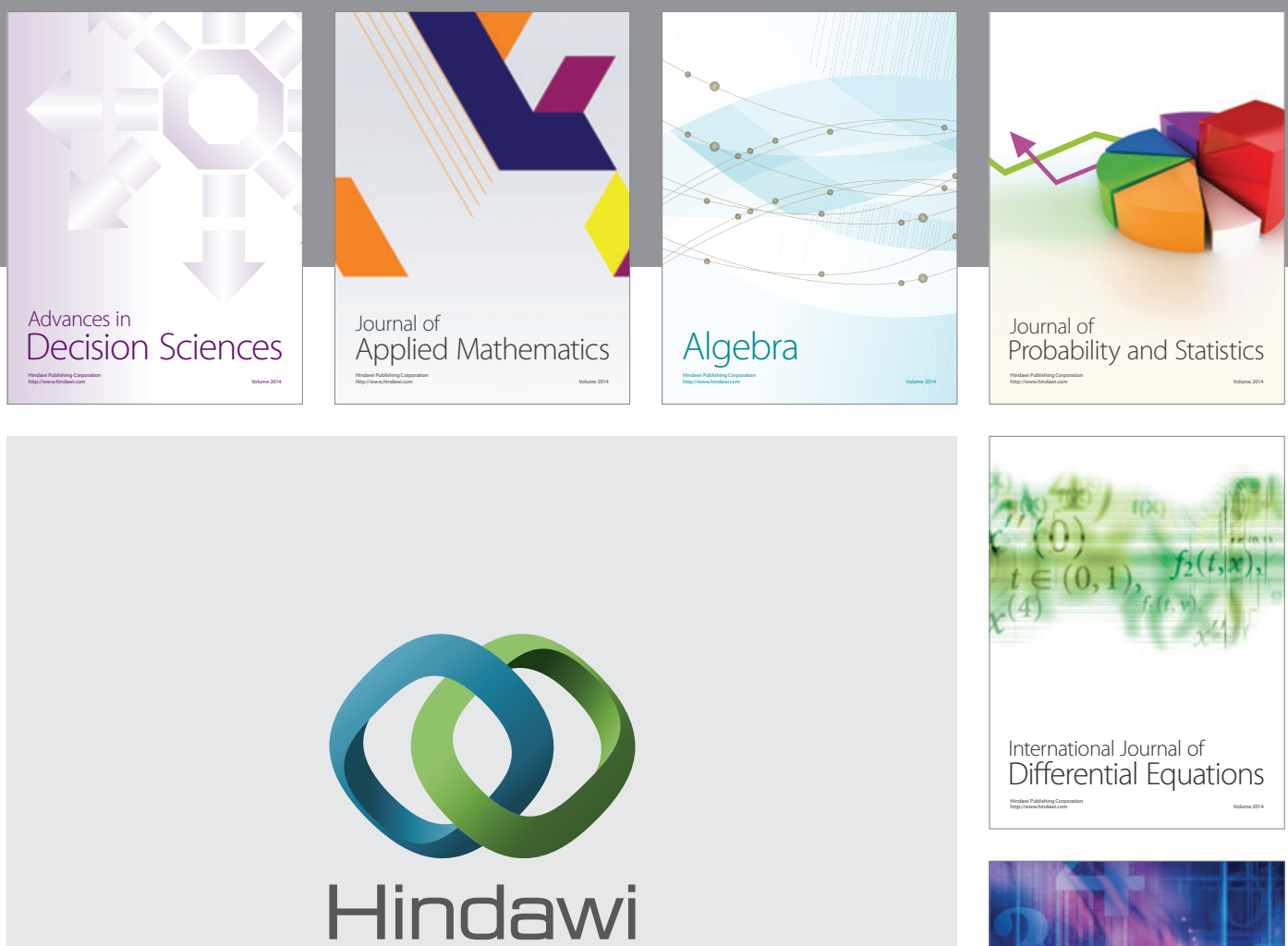

Submit your manuscripts at http://www.hindawi.com
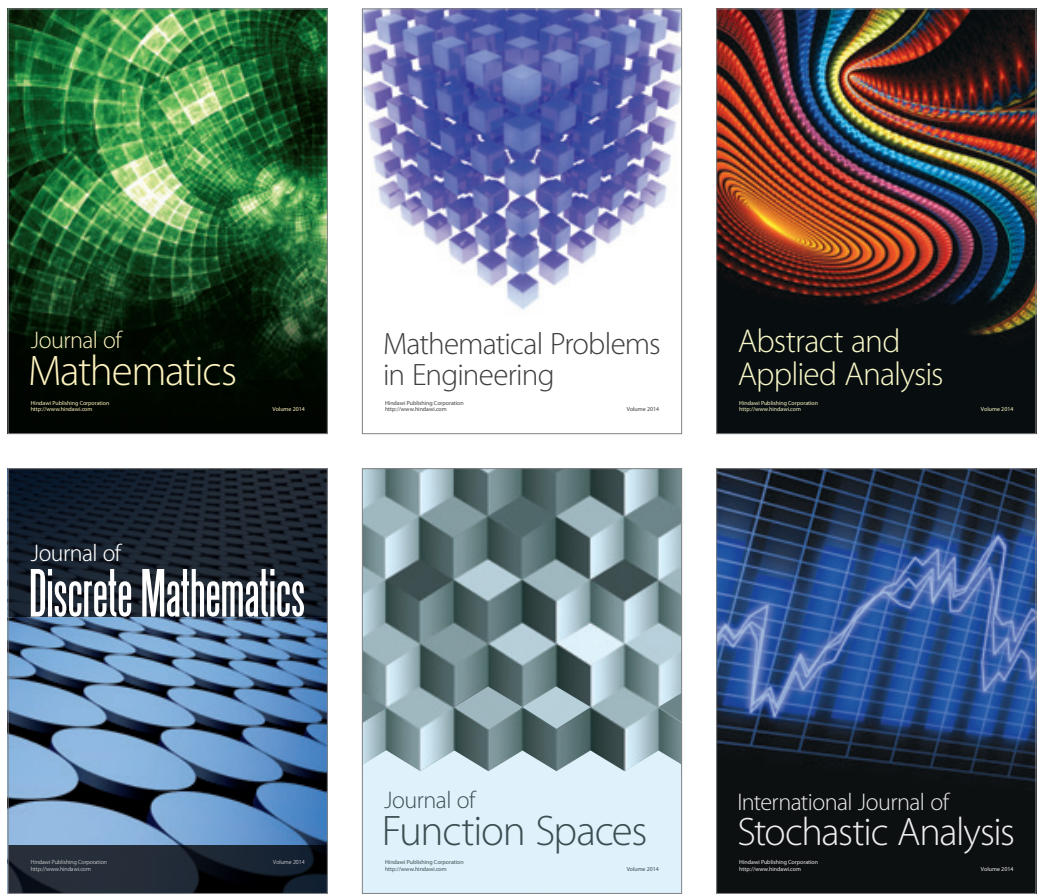

Journal of

Function Spaces

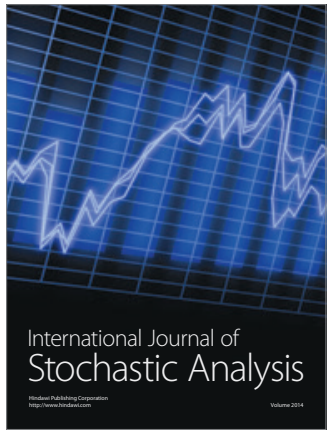

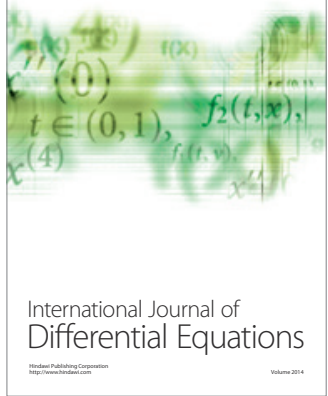
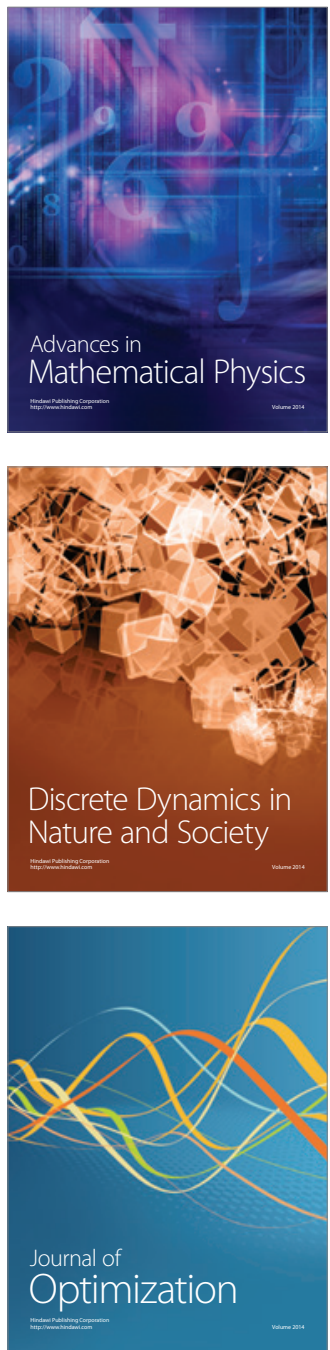